Full length article

\title{
The influence of social cues in persuasive social robots on psychological reactance and compliance
}

\author{
Aimi Shazwani Ghazali ${ }^{\mathrm{a}, \mathrm{b}, *}$, Jaap Ham ${ }^{\mathrm{c}}$, Emilia Barakova ${ }^{\mathrm{a}}$, Panos Markopoulos ${ }^{\mathrm{a}}$ \\ a Eindhoven University of Technology, Department of Industrial Design, 5612 AZ Eindhoven, The Netherlands \\ ${ }^{b}$ International Islamic University Malaysia, Department of Mechatronics Engineering, Jln. Gombak, 53100 Kuala Lumpur, Malaysia \\ ${ }^{c}$ Eindhoven University of Technology, Department of Industrial Engineering \& Innovation Sciences, 5612 AZ Eindhoven, The Netherlands
}

\section{A R T I C L E IN F O}

\section{Keywords:}

Persuasion

Human-robot interaction

Social cues

Psychological involvement

Psychological reactance

Compliance

\begin{abstract}
A B S T R A C T
People can react negatively to persuasive attempts experiencing reactance, which gives rise to negative feelings and thoughts and may reduce compliance. This research examines social responses towards persuasive social agents. We present a laboratory experiment which assessed reactance and compliance to persuasive attempts delivered by an artificial (non-robotic) social agent, a social robot with minimal social cues (human-like face with speech output and blinking eyes), and a social robot with enhanced social cues (human-like face with head movement, facial expression, affective intonation of speech output). Our results suggest that a social robot presenting more social cues will cause higher reactance and this effect is stronger when the user feels involved in the task at hand.
\end{abstract}

\section{Introduction}

The use of robots as a technology to support attitudes and behavior changes is attracting a lot of interest from researchers (Agrawal \& Williams, 2017; Lopez, Ccasane, Paredes, \& Cuellar, 2017). In enhancing the persuasiveness of such artificial social robots and the emerging human-robot interaction experiences, it is essential to understand how people perceive diverse attitudes and social behaviors of robots. Ham, Cuijpers, and Cabibihan (2015) claimed that the persuasiveness of a storytelling robot could be increased by adding social cues like gazing and gestures. Social cues such as movement of robot's head to track human's motions and maintaining eye contact throughout a conversation have been shown to increase feelings of immersion in a task (Bailenson, Blascovich, Beall, \& Loomis, 2006; Li, 2013). The earlier research studied to what extent social robots should portray social characteristics to elicit perceived social agency to be able to make use of user's social psychological responses towards the robot (Chetouani, Boucenna, Chaby, Plaza, \& Cohen, 2017; Choi, Kornfield, Takayama, \& Mutlu, 2017; Thimmesch-Gill, Harder, \& Koutstaal, 2017). Some theories like the media equation hypothesis (Martin, 1997) suppose that basic social characteristics suffice to elicit social responses, and earlier research confirms this notion (Chidambaram, Chiang, \& Mutlu, 2012; Roubroeks, Midden, \& Ham, 2009). Relatedly, the social-cues hypothesis (Louwerse, Graesser, Lu, \& Mitchell, 2005) explained that adding human features as social cues on the robot like facial expression, voice, and physical presentation could enhance the chance for a human to perceive the technology more positively. This hypothesis was also supported by findings in several studies (Andrist, Spannan, \& Mutlu, 2013; Cooney, Dignam, \& Brady, 2015; Eyssel \& Hegel, 2012).

However, when people are subjected to strong persuasive attempts, they may respond negatively towards the attempt, with a behavior that is known as psychological reactance. Psychological reactance is defined as an action or act of the doer that is different from their original intention because of persuasion activities that can provoke feelings of anger and negative cognitions (Dillard \& Shen, 2005). It is a motivational response to the loss of freedom or threatened with reduction of alternatives (Brehm \& Brehm, 2013; Brehm, 1972). Psychological reactance can lead to irregular behaviors in restoring the freedom in making a decision. People may not comply and even will do something that is opposite than what they are asked to do. Earlier research (Dillard \& Shen, 2005; Lee, Lee, \& Hwang, 2014; Rains \& Turner, 2007) has shown that psychological reactance can be measured using questionnaires. Experimental studies have attempted to identify the cause of reactance and how human behave to portray their reaction towards the reactance. For example, earlier researcher has shown that forceful language in persuasive communications in a health campaign can be a source of reactance (Quick \& Considine, 2008). An experimental study (Roubroeks, Ham, \& Midden, 2011) found that people experience

\footnotetext{
* Corresponding author. Eindhoven University of Technology, Department of Industrial Design, 5612 AZ Eindhoven, The Netherlands

E-mail addresses: A.S.Ghazali@tue.nl, aimighazali@iium.edu.my (A.S. Ghazali), J.R.C.Ham@tue.nl (J. Ham), E.I.Barakova@tue.nl (E. Barakova), P.Markopoulos@tue.nl (P. Markopoulos).
} 
higher psychological reactance when persuasive text messages are accompanied by a still picture of the persuasive agent, or a short film-clip showing the persuader deliver this message, concluding that stronger social agency of the persuasive source can lead to higher psychological reactance. As the interest in applications of artificial agents and especially social robots in care scenarios, it is important to understand how to design these agents to be more effective in their persuasive communication and to avoid that they evoke negative feelings to users. Specifically, it is important to understand the impact of social cues that can be implemented in such artificial agents upon reactance.

In this research, we aim to evaluate the effect of social cues of an agent upon reactance and compliance as well as the level of involvement of a person with the issue at hand. It can be expected that when an agent limits a person's freedom about an issue they are not involved in, reactance may be lower or not occur, but when a person's freedom is limited about an issue in which that person is strongly involved, they may experience stronger reactance. Several studies have investigated the effects of involvement towards human's psychophysiological responses in an interactive game (Lim \& Reeves, 2009) like engagement level between gameplays with avatars or computer agents (Lim \& Reeves, 2010) and persuasion (Johnson \& Eagly, 1989; Oreg \& Sverdlik, 2014). From those studies, it can be concluded that in high-involvement situations, the chances for successful persuasion activities are low, and that in such situations people may easily experience reactance. In contrast, in low-involvement situations, chances for successful persuasion might be higher, but in such situations reactance is not very likely to occur. Nevertheless, earlier research has not yet examined the effect of involvement upon of reactance.

In line with social agency theory (Atkinson, Mayer, \& Merrill, 2005), people will be more socially responsive to the agent that has more social cues. Unintuitive and in contrast to earlier reactance studies (e.g. Roubroeks et al., 2009), a recent experiment reported by Ghazali, Ham, Barakova, and Markopoulos (2017) found that robotic agents evoked less reactant responses when using unpleasant language in persuasive messages. That is, the reactance towards a robotic agent that used forceful language to persuade people was lower when the robotic agent displayed some social cues. Thus, this earlier study did not show that people respond in more social ways (i.e., show more reactance) when a social robot displays more social cues in delivering the forceful persuasive message. Nevertheless, the external validity of that experiment can be criticized as the decision that experimental participants had to make pertained to an artificial task with little at stake for them. Specifically, the experimental task was to decide upon the constitution of a drink for an imaginary alien, a choice behavior for which the participants did not care about. The authors claimed that it was done to avoid confounding effects of psychological involvement with the task at hand. However, it leaves the question open whether the results can be replicated in case the participants have higher involvement with the given tasks.

Thus, this paper builds on and extends the study of Ghazali et al. (2017) which compared social agents that were endowed with three different levels of social cues. It aims to address the limitations of that study discussed above and to consolidate current understanding of the effects of social cues on social responses as suggested by social agency theory (Atkinson et al., 2005). We report an experiment that compared the situations of high and low psychological involvements in persuasion activity in different social agency conditions. The following sections motivate the method and describe the results of our study. We conclude with a discussion regarding the implications of our findings for the field of persuasion in human-robot interaction applications and research on psychological reactance.

\subsection{The current study}

The experimental set up involved a human-agent interaction in which the participants were asked to make decisions in a fantasy game environment, similar to that of Ghazali et al. (2017). Participants were required to make an initial selection of a drink, after which an artificial agent would attempt to convince them to modify their choice. High controlling language was used by the social agent in conveying the advice throughout the study. This was done to obtain higher chances of compliance in persuasive attempts as reported in previous research (Ghazali et al., 2017). The experiment aimed to test the following two hypotheses:

H1. Participants in the high psychological involvement game will experience higher psychological reactance than those who receive the same advice in a low psychological involvement game, especially when the advisor had higher social agency.

H2. Participants in the low psychological involvement game will be more compliant to change their final decisions when being advised by an agent with a high social agency compared to the participants with high psychological involvement receiving feedback by the same agent.

\section{Materials and methods}

This study was carried out in accordance with the recommendations of Code of Ethics of the NIP (Nederlands Instituut Voor Psychologen Dutch Institute for Psychologists) and the research group on HumanTechnology Interaction at Eindhoven University of Technology. All subjects gave written informed consent in accordance with the Declaration of Helsinki. This study was reviewed and approved by the Human-Technology Interaction ethics board at Eindhoven University of Technology.

\subsection{Participants and design}

Sixty participants were recruited as volunteers from a local participant database with ages ranging from 18 to 37 years old (41 males and 19 females; age $M=23.98, S D=3.71$ ). A between-subjects experimental design was used in this study to avoid the carry-over effects as found in within-subjects design study (Yang et al., 2017). The participants were divided into six groups randomly assigned to a particular level of social agency (low vs. medium vs. high) and psychological involvement (low vs. high). Each participant received a $€ 10$ voucher as a token of appreciation at the end of the session which lasted $40 \mathrm{~min}$ on average.

\subsection{Manipulations}

\subsubsection{Manipulation of social agency}

The manipulation of social agency of the advisor in this experiment was based on the number of social cues portrayed as (1) low social agency: absence of a robot - the advice was displayed on a screen as an advisory-text (2) medium social agency: a robot with a human-like face that spoke with monotone voice and showing minimal nonverbal cues (blinking eyes) (3) high social agency: the robot gave advice using several verbal and nonverbal social cues including head movements (e.g., nodding the head), eye expressions (e.g., looking away indicates the robot was thinking) and emotional intonation in the voice. As in Ghazali et al. (2017), a Socibot robot was used in medium and high social agency conditions. SociBot is a desktop robot that displays an animated face through back projection and offering some built -in functionalities such as move its head, track a user movements etc. The robot is also equipped with lip-synced speech output and can give the impression of maintaining eye contact with the participants throughout the experimental session. It was given the facial image of a man with light brown skin color tone and hazel eyes. Various facial expressions were displayed by the robot in the high social agency condition only. An overview of the social agency manipulation is shown in Fig. 1.

The robot was operated by the experimenter using Wizard of $\mathrm{Oz}$ 


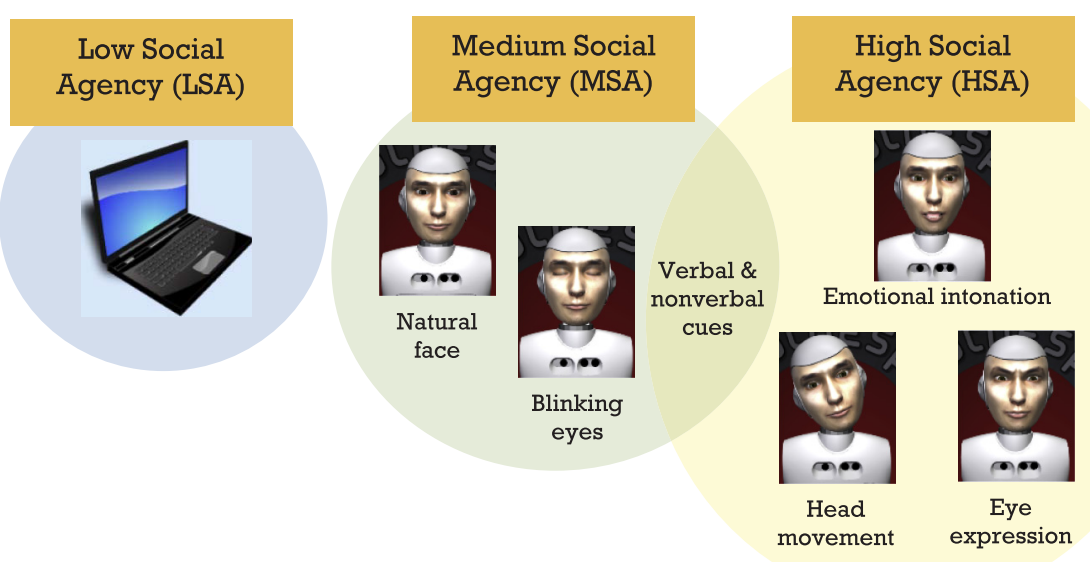

Fig. 1. Manipulation of social agency conditions.

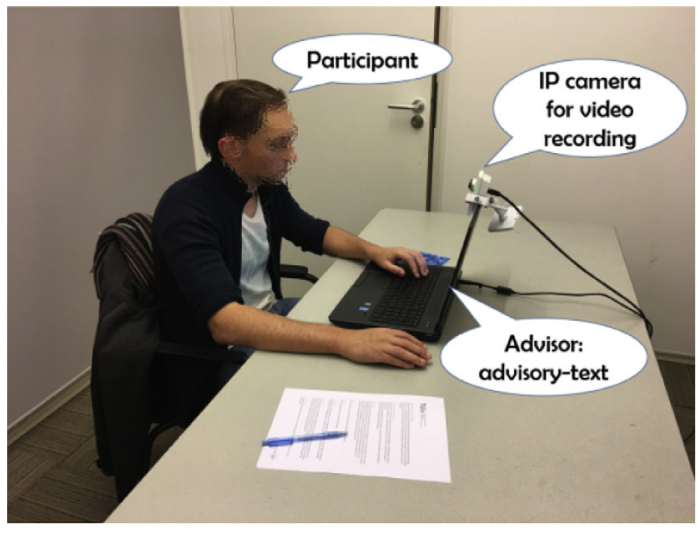

(a)

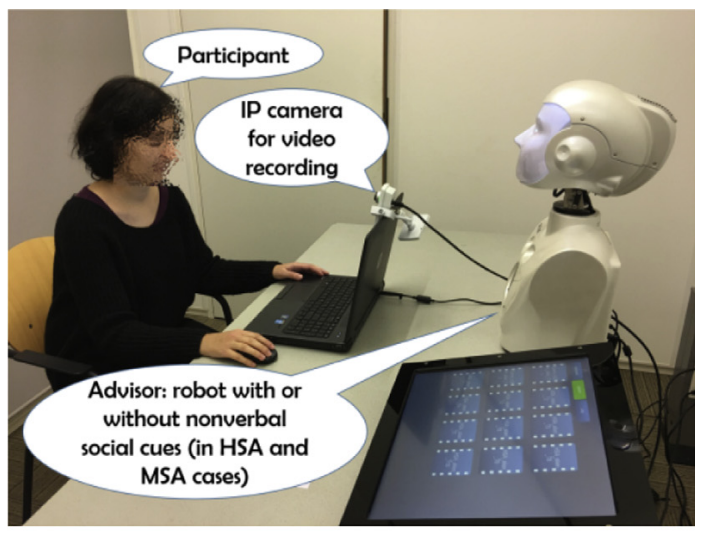

(b)

Fig. 2. Experimental set ups for social agency manipulation: (a) Low Social Agency (LSA) (b) Medium Social Agency (MSA) and High Social Agency (HSA).

method in choosing pre-selected persuasive messages at suitable moments during the experiment. Synthetic speech output was played by the robot's speaker. In contrast, for the low social agency condition, the participants needed to read the advice as it was displayed on a laptop screen. Fig. 2 illustrates the experimental set ups used.

\subsubsection{Manipulation of psychological involvement}

As mentioned already, participants were exposed to either of two levels of psychological involvement which we label as low and high, based on the degree of perceived relevance of the tasks to the participant. In the low psychological involvement game, the participants were asked to create a drink for an alien while participants in high psychological involvement game were required to create a drink for themselves (to drink after the experiment). The examples of high controlling, forceful language advice for both psychological involvement level provided by the respective social agent as follows (a) Low psychological involvement: "What a bad choice. The constitution of the drink you chose before was very bad for the alien's health condition. You must serve other drink to the alien. I am sure the alien will love it!" (b) High psychological involvement: "What a bad choice. The constitution of the drink you chose before was very bad for your health condition. You must choose other drink for yourself. I am sure you will love it!"

\subsection{Task}

We selected a game as a medium to deliver the experimental task since games are engaging and can keep the players' concentration high and prevent boredom during the 25 min of the experimental session
(Jacobs, 2016; Lawson \& Semwal, 2016). The task for this study is based on an online game called 'Smoothie Maker: Creation Station'. The theme of the game (e.g., creating a drink) was carefully chosen to ensure that the source of the reactance experienced by participants solely from the social agent, instead of dense and stressful topics like political views, healthy lifestyle, etc. for which very variable levels of involvement could be expected amongst participants.

In the original online game, the participants make several decisions regarding e.g. which fruit they prefer or which straw they find attractive to be used to drink the smoothie. We reproduce a similar game's theme for this experiment called 'Beverages Creation Station' using Matlab software. In adapting the original game concept for this experiment, several changes have been made. First, the role of the social agent was to advise the participants after each smoothie selection had been created. Second, the choices given in each task were different from the original game to fit the participants' age range and to ensure the anonymity of choices. Third, the game with a low level of psychological involvement was added to manipulate the involvement factor as only high psychological involvement game was presented in the original game. Also, the game consisted of multiple tasks to provide an extended interaction between the participants and the social agent. Ten tasks needed to be completed by the participants in each session. The tasks consisted in three, four or ten multiple-choice questions depending on the preset answers by the experimenter. The difficulty level of choices remained constant during the game. The background sound from the original game also was removed to avoid distracting participants during the experiment.

The social agent used high controlling language (unpleasant and 
pushy language) all the time in expressing the persuasive advice towards the participants to change their initial selection to other choices as their final answer. Although the psychological involvement was manipulated in this experiment, the core concept of the advice made by the social agent was kept as ambiguous as possible. An example of the recommendation in low social agency session for high psychological involvement game was "What a childish selection! You cannot even finish up the whole drinks if you choose a big container so in the end that delicious drink will just be thrown away. It is a waste. However, if you choose a small container, you need to pay some amount of money to get other drinks. Just choose another container that contained a right amount of drinks which fit your tummy appropriately. Do not be too greedy, but at the same time, do not be too absurd". Whatever choice participants would make the advisor would not agree and would try to persuade them to change it. For example, participants could choose between two responses to the message above: keep their initial selection of the container size (ignore the advice), or change their mind and select a container with a different size in following the advice. The participants were also reminded that the social agent has a similar level of social power with the participants in making a decision. Specifically, participants were told that "You are free either to follow or to ignore the advice given. There will be no right and wrong answers in this game".

\subsection{Procedure}

The experiment took place in a dedicated room. Arriving participants provided consent and demographic information before they were introduced to the social agent corresponding to the experimental condition they were assigned to. A SociBot was placed in front of participants assigned to the medium and the high social agency conditions; during the demonstration session they were shown how the SociBot delivers advice. For the low social agency condition session, there was no robot present, and the advice would come in the form of advisorytext on a laptop screen. The experimenter demonstrated how to play the game using the 'Demonstration' Graphical User Interface and left the room when the participants had no more questions about it. The 'Demonstration' user interface was the same as used during the session.

Participants were reminded about the psychological involvement level assigned to them in each task. Let's say they were in the low psychological involvement condition where a drink should be made for the alien; then a reminder would be presented on the laptop screen displaying the game: 'Please remember! The drink is for the ALIEN, not for $Y O U$ '. In contrast, in the high psychological involvement condition, the participants would be prompted with a message reading as follows: 'Please remember! The drink is for YOU, not for OTHERS'.

Finally, after finishing the game and answering the questionnaires required in Google form, the experimenter would return to the room and present a token of appreciation to each participant. The session officially finished after the experimenter debriefed the participants.

\subsection{Measures}

\subsubsection{Psychological reactance}

We used two questionnaires (intertwined model of negative cognitions and feelings of anger) to measure the psychological reactance experienced by the participants (Dillard \& Shen, 2005; Quick \& Stephenson, 2007). Specifically, participants were asked to indicate their level of irritation, angriness, annoyance, and aggravation after playing the game on a 5-level Likert Scale ranging from (1) completely disagree to (5) completely agree. They were also required to report what thoughts they had while playing the game and to label these as negative, positive or neutral. Then the negative cognitions were counted according to the procedure proposed by Dillard and Shen (2005). After that, the negative cognitions score was submitted as one of the components in psychological reactance measure in percentage form (Roubroeks, Midden, \& Ham, 2011).

\subsubsection{Compliance}

The compliance of participants was measured as the number of times participants changed their initial decision to comply with the agent's advice as in Ghazali et al. (2017). Participants had ten choice moments during the experimental session. In case the initial choice was the same as the final choice, then the participants would not get any compliance point for that particular task. In contrast, if the initial and final choices were inconsistent, it showed that the participants were successfully being persuaded by the advisor to change their choice and they would be awarded 1-point for that particular task. E.g., if a particular participant would follow social agent's advice and changed his/ her final choice as instructed for task number 2, 3, 4, 5, 8 and 10 and was incompliant for the other four tasks; then he/she would be given the compliance score of 6 .

\subsubsection{Other measures}

Apart from psychological reactance and compliance measures, two manipulation checks were done to assess whether participants perceived the advice from different social agents as threatening and to check whether the manipulation of psychological involvement affects the level of immersion towards the game created.

Walter and Lopez (2008) defined perceived threat to autonomy as the degree to which a person believes the threat could control the condition or content of his/her autonomy in making a selection. Since the manipulation of social agency may be associated with autonomy in decision making, we want to check whether the participants are likely to perceive the persuasive attempts by different level of social agency as a threat. The perceived threat to autonomy measure consisted of four statements which were: 'The advisor restricted my autonomy to choose what I want to serve', 'The advisor tried to manipulate me', 'The advisor tried to make a decision for me' and 'The advisor tried to pressure me'. Participants could answer on a 5-point Likert Scale ranging from (1) completely disagree to (5) completely agree.

To check whether the manipulation of psychological involvement was successful, an adaptation of two different questionnaires developed in earlier studies (Mittal, 1989; van Wijngaarden et al., 2000) was made for evaluating how strong the associated immersion was experienced by the participants during the game. Participants were asked to answer five immersion questions about the degree of importance, concern, involvement, care, and responsibleness towards the decision taken about making a tasty drink. Participants could answer on a 5-point Likert Scale ranging from (1) completely disagree to (5) completely agree.

\section{Results}

Statistical analysis was carried out using IBM SPSS version 23 . The results of the analysis are presented in two parts: manipulation check and hypothesis test.

\subsection{Manipulation check}

ANOVA tests were conducted to check whether the variation of social agency and psychological involvement caused differences in the level of perceived threat to autonomy in making decisions and the level of immersion towards the game.

\subsubsection{Perceived threat to autonomy}

First, we checked whether the participants perceived the manipulation of social agency as a threat to their autonomy in making decision. No significant effect of the social agency manipulation was found on perceived threat to autonomy, $F(2,58)=0.88, p=0.42$. This finding indicates that the level of social agency of the agent did not influence the extent to which participants felt threatened.

In addition, the main effect of psychological involvement on perceived threat to autonomy was significant, $F(1,59)=4.26, p=0.04$, 
with low psychological involvement: $M=3.90(S D=0.55)$ and high psychological involvement: $M=3.58(S D=0.64)$. Results show that the participants in low psychological involvement game (making alien's drink) perceived the advice given by the social agent as a threat, higher than the participants in high psychological involvement's game (creating own drink).

\subsubsection{Immersion}

Second, we checked whether the manipulation of psychological involvement was successful. Results indicate that the psychological involvement had a significant contribution to the level of immersion, $F$ $(1,59)=3.87, p=0.05$, low psychological involvement: $M=3.69$ $(S D=0.83)$ and high psychological involvement: $M=4.07$ $(S D=0.63)$. These results show that the participants in high psychological involvement game (creating one's own drink) were much more immersed in the game compared to other participants who were in low psychological involvement game (creating alien's drink). This result confirms that ostensibly making a drink for an alien versus oneself was an effective manipulation of psychological involvement.

Additionally, no significant main effect of social agency was found on the level of immersion, $F(1,59)=3.87, p=0.60$ (n.s). Results indicate that the level of social agency did not influence the level of immersion towards the game.

\subsection{Hypothesis test}

\subsubsection{Hypothesis 1}

Repeated measures of psychological reactance consisting of two components of within-subject factors (feelings of anger and negative cognitions $^{1}$ ) were used to investigate the first hypothesis. First, a Pearson product-moment correlation test between feelings of anger and the rate of self-reported negative cognitions demonstrated that there was a weak correlation between these two variables $(r=0.16, n=60$, $p=0.22(n . s)$ ). This is in line with earlier research (Dillard \& Shen, 2005), as they measure two aspects of the same phenomenon that cannot be completely separated from each other.

To test hypothesis 1, a repeated measures Analysis of Variance (ANOVA) test was run with social agency and psychological involvement as the independent variables and psychological reactance score as the dependent variable. The two components of psychological reactance were treated as a repeated measures factor in this analysis.

As a result, the manipulation of psychological involvement level was found to have a significant effect upon the measured psychological reactance $\left(F(1,48)=4.315, p=0.04\right.$, partial $\left.\eta^{2}=0.08\right){ }^{2}$ Besides, the social agency level also had a significant influence on the psychological reactance, $F(2,48)=8.20, p=0.001$, partial $\eta^{2}=0.26$. More importantly, there was a significant interaction between social agency and psychological involvement manipulations on psychological reactance, $F$ $(2,48)=4.14, p=0.02$, partial $\eta^{2}=0.16$ (see Fig. 3).

Several conclusions can be drawn from this analysis. First, concerning the psychological involvement, reactance recorded in making own drink $(M=13.45, S D=9.75)$ was higher than in making alien's drink $(M=8.16, S D=9.97)$, especially when the appointed advisor was a robot in high social agency condition. Meanwhile, there was a similar reactance score for the participants in low social agency

\footnotetext{
${ }^{1}$ The score for feelings of anger showed no extreme outlier and was normally distributed. However, the score for negative cognitions was not normally distributed. We proceeded to use the repeated measures ANOVA for testing the first hypothesis because (in line with statistical insights (Glass, Peckham, \& Sanders, 1972; Harwell, Rubinstein, Hayes, \& Olds, 1992; Lix, Keselman, \& Keselman, 1996)) the score for negative cognitions was distributed similarly (non-normally) in all of the $3 \times 2$ cells, and because ANOVAs are considered fairly "robust" to deviations from normality.

${ }^{2}$ In the Hypothesis 1 analysis, we use gender as an additional predictor, because we assume it to explain variance of the manipulations of social agency and psychological involvements. However, since we do not have any hypothesis about the effects of gender, we do not report its effects.
}

condition for both making their own and the alien's drinks. Second, with respect to the level of social agency, participants in the high social agency conditions $(M=17.64, S D=10.20)$ experienced the highest reactance, followed by the low social agency condition $(M=10.12$, $S D=9.17)$ and the lowest reactance was in medium social agency condition the $(M=4.65, S D=10.20)$. Fig. 3 also indicates that participants who made their own drink while interacting with a high social agency advisor had the highest reactance. The lowest reactance was experienced by participants in the medium social agency condition. Importantly, there was a clear increment of psychological reactance level (the differences of psychological reactance mean values) with the increment of social agency's level.

An exploratory analysis examined the individual effects of psychological reactance score (feelings of anger and negative cognitions as two separate dependent variables) resulting from the manipulations of social agency and psychological involvement using a two-way ANOVA test. A significant interaction was found between social agency and psychological involvement for the negative cognitions score, $F$ $(2,48)=4.35, p=0.02$, partial $\eta^{2}=0.15$. Also, there was a statistically significant difference in the negative cognitions score between low, medium and high social agency conditions for a high psychological involvement game, $F(2,48)=10.43, p=0.001$, partial $\eta^{2}=0.30$. However, the simple main effect of the social agency on the mean negative cognitions score for those who participated in the low psychological involvement game was not statistically significant, $F$ $(2,48)=1.61, p=0.21$, partial $\eta^{2}=0.06$. Overall, the mean of negative cognitions in the high psychological involvement's game $(M=22$, $S D=23.1$ ) was significantly higher than in the low psychological involvement game $(M=16.67, S D=16.67), F(1,48)=4.39, p=0.04$, partial $\eta^{2}=0.08$.

As for feelings of anger, there was no statistically significant interaction between social agency and psychological involvement, $F$ $(2,48)=0.22, p=0.81$, partial $\eta^{2}=0.01$. We also found no significant main influence of social agency and psychological involvement on the reported feelings of anger, $F(2,48)=0.03, p=0.98$ and $F$ $(2,48)=0.03, p=0.86$ respectively. As such, these results demonstrate that the lowest feelings of anger were experienced by participants in the low psychological involvement game while interacting with advisor in with low social agency condition $(M=3.10, S D=0.74)$. On the other hand, the highest feelings of anger recorded by participants playing the high psychological involvement game in the low social agency condition $(M=3.25, S D=1.21)$.

\subsubsection{Hypothesis 2}

The second hypothesis stated that the participants who were advised by the agent with high social agency, especially those who played the low psychological involvement game, would be more compliant in changing their final decisions compared to the participants playing the high psychological involvement game. To test the effect of both social agency and psychological involvement manipulations on compliance score, a two-way ANOVA test was conducted. The result revealed that there was no significant interaction of social agency and psychological involvement manipulations on the compliance, $F(2,54)=0.42$, $p=0.66$, partial $\eta^{2}=0.02$. It is interesting to note that the relationship was statistically significant when the manipulation of psychological involvement was the only independent variable used with the compliance score as the dependent variable, $F(2,54)=35.43, p<0.001$, partial $\eta^{2}=0.40$.

The pattern of compliance (summation of all task's score) based on the manipulations of social agency and psychological involvement can be observed in Fig. 4. By comparing all conditions, participants who were advised by an agent with high social agency in high psychological involvement game showed the highest noncompliance by neglecting most of the given advice. Univariate tests reveal a significant simple effect of psychological involvement within each level combination of social agency manipulation towards compliance score. This test 


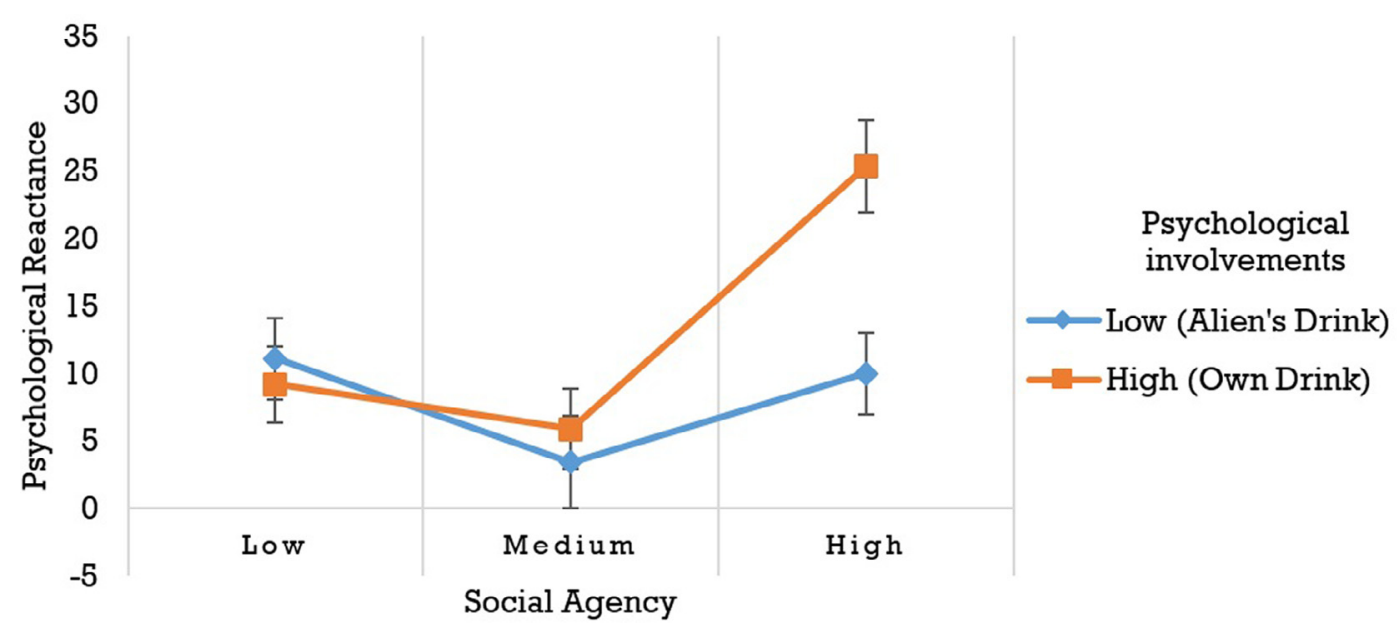

Fig. 3. Mean and standard error of psychological reactance scores by social agency (low vs. medium vs. high) and psychological involvement (low vs. high). Participants in the low psychological involvement condition reported lower reactance compared to participants in high psychological involvement condition (independent of the social agency). Participants in the medium social agency condition reported lower psychological reactance compared to participants in the low and high social agency conditions. There was a significant interaction effect of social agency and psychological involvement on psychological reactance.

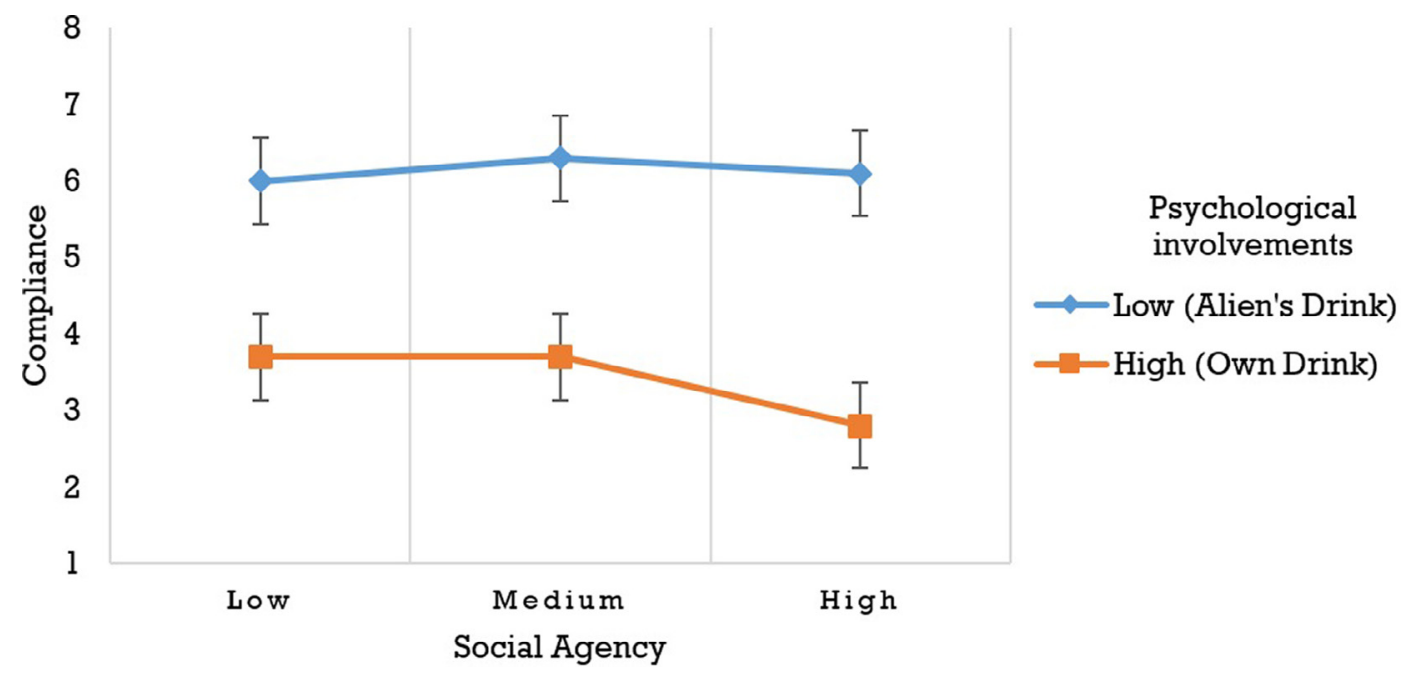

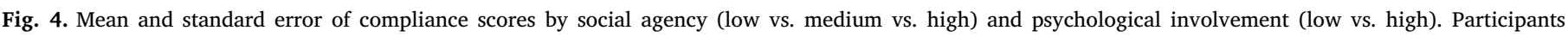

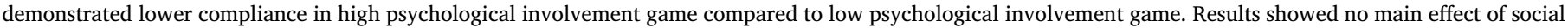
agency, and no interaction effect between social agency and psychological involvement on compliance.

demonstrates a statistically significant difference in compliance scores between low psychological involvement and high psychological involvement games onto compliance using between-subject advisor with low social agency $F(1,54)=8.36, p=0.01$, partial $\eta^{2}=0.13$, medium social agency $F(1,54)=10.69, p=0.002$, partial $\eta^{2}=0.17$ and high social agency $F(1,54)=17.22, p<0.001$, partial $\eta^{2}=0.24$.

Regarding the manipulation of social agency, although there were only small differences in compliance scores between the three social agency levels (low vs. medium vs. high social agency), the participants in the medium social agency condition $(M=5.00, S D=2.47)$ showed the highest cumulative compliance score. Whereas, participants that interacted with the robot with maximal social cues in high social agency condition were the least compliant $(M=4.45, S D=2.04)$. This result is in agreement with the reactance measured in the first hypothesis, in which the medium social agency's participants experienced the lowest reactance compared to other social agency conditions.

Regarding psychological involvement, the participants who were making their own drink (high psychological involvement) refused to follow the advice more often $(M=3.40, S D=1.54$, total compliance score of 102) than those making the alien's drink $(M=6.13, S D=1.92$, total compliance score of 168). Additionally, there was no consistent pattern to show that the compliance changes over time (based on the task number) for both the manipulations of social agency and psychological involvement. Although the social agent kept on disagreeing with the participants' initial choice at every single decision point, it shows that compliance score was not influenced by the behavior of the social agent over the time. In other words, the impact of the advisor on the decisions made by the participants did not change over time.

\section{Discussion}

The primary purpose of this study was to investigate human's social responses (psychological reactance and compliance) on several social agency conditions in persuasion activity. In line with social agency theory (Atkinson et al., 2005), we expected that social agents with more social cues would elicit higher psychological reactance compared agents with minimal or no social cues. This study also compared the difference in social responses experienced by humans when they were put in the situation of either high or low psychological involvement.

Hypothesis $\mathrm{H} 1$ was confirmed only partly. We found that as the level of social agency and psychological involvement increased, psychological reactance would increase as well, in line with previous 
research (Roubroeks et al., 2011; Roubroeks et al., 2009). Contrary to our expectations, an agent with medium social agency, (i.e., with minimal social cues) provoked the lowest psychological reactance in both psychological involvement conditions (refer to Fig. 3). We assumed that the high social agency advisor evokes the highest reactance because of the forceful voice tone and pressure portrayed by the robot that attempted to convince participants into changing their choices for each task. A possible explanation why the psychological reactance in medium social agency condition was lower than in the low social agency is that in the medium social agency case, the absence of facial expressions and the unemotional intonation of the robot, could be perceived as less forceful way to deliver advice, compared to text which could be assumed to be delivered forcefully. This result can be explained by the finding that some of the participants indicated that they had experienced that the advice was delivered in a forceful tone, high pitch which may have caused higher reactance to happen (compared to medium social agency condition). Apart from the low social agency condition, the psychological reactance in the low psychological involvement game was always lower than in high psychological involvement game, as the participants would experience higher reactance when they were pushed to change the choice of their own drink. There could be two explanations for this: participants may be more receptive to advise in the low psychological involvement condition as they do not know what drink aliens like best or because they do not care as much for what drink the alien will have. However, as they know more what they like to have compared to the persuader (the social agent) in high psychological involvement game, they felt more anger and had more negative cognitions towards the agent when they were pushed to change their choices.

The second hypothesis suggested that the participants that made own drink in the game (high psychological involvement) would be less compliant than those in the low psychological involvement game, as the advice was delivered by an agent with lower social agency. Results demonstrate that the manipulation of psychological involvement had a statistically significant effect upon the compliance score, but failed to reveal any such effects with the manipulation of social agency. By referring to Fig. 4, it can be observed that the participants prefer to follow the advice for the alien's drink (regardless the level of social agency) perhaps because they believed that the advisor knew the alien's preference better than themselves. In contrast, when the participants were asked to create their own drink, as they were very sure of what they would want to have; advice from the social agent was always disregarded. Thus, the compliance recorded during high psychological involvement game was always lower than low psychological involvement game.

The most important finding emerging from this study is that the differences of psychological reactance (discussed in Hypothesis 1) and compliance (discussed in Hypothesis 2) scores between low and high psychological involvement games increased with the addition of social cues in the agents (see Figs. 3 and 4 respectively). It shows that social cues displayed in the higher social agency condition influence people to interpret the agent as a real human during the interaction (Martin, 1997). This finding also is in agreement with social agency theory (Atkinson et al., 2005) that argues that the more social characteristics a robot can display, the higher the social responses that humans will exhibit during human-robot interaction.

\subsection{Limitations and suggestions for further researches}

The current experimental study can be improved in several ways. First, only two social responses were examined in this experiment: psychological reactance and compliance. Despite these promising results, questions remain about other social responses like trust towards the persuasion activity. Additional evaluation of other social responses shall be added to enrich the understanding of using a robot as a persuader. Second, there are still many unanswered questions about the human acceptance of technology with agents that can lead to future research in understanding the relative impact of social cues used by the persuasive robotic agent and especially the extent to which they evoke psychological reactance.

\section{Declaration of interest}

None.

\section{Acknowledgements}

The authors wish to acknowledge the participants who took part in this study. This work was funded by Malaysia Ministry of Higher Education and International Islamic University Malaysia. Also, Jaap Ham's contributions were partly supported by project Multimedia Authoring \& Management using your Eyes \& Mind (MAMEM) that has received funding from the European Union's Horizon 2020 research and innovation program under grant agreement number: 644780 .

\section{References}

Agrawal, S., \& Williams, M. A. (2017). Robot authority and human obedience: A study of human behaviour using a robot security guard. Proceedings of the companion of the 2017 ACM/IEEE international conference on human-robot interaction (pp. 57-58). ACM. http://dx.doi.org/10.1145/3029798.3038387.

Andrist, S., Spannan, E., \& Mutlu, B. (2013). Rhetorical robots: Making robots more effective speakers using linguistic cues of expertise. Proceedings of the 8th ACM/IEEE international conference on human-robot interaction (pp. 341-348). IEEE Press.

Atkinson, R. K., Mayer, R. E., \& Merrill, M. M. (2005). Fostering social agency in multimedia learning: Examining the impact of an animated agent's voice. Contemporary Educational Psychology, 30(1), 117-139. http://dx.doi.org/10.1016/j.cedpsych.2004. 07.001.

Bailenson, J. N., Blascovich, J., Beall, A. C., \& Loomis, J. M. (2006). Equilibrium theory revisited: Mutual gaze and personal space in virtual environments. Equilibrium, 10(6), 583-598. http://dx.doi.org/10.1162/105474601753272844.

Brehm, J. W. (1972). Responses to the loss of freedom: A theory of psychological reactance. Morristown, NJ: General Learning Press.

Brehm, S. S., \& Brehm, J. W. (2013). Psychological reactance: A theory of freedom and control. New York: Academic Press.

Chetouani, M., Boucenna, S., Chaby, L., Plaza, M., \& Cohen, D. (2017). 28 social signal processing and socially assistive robotics in developmental disorders. Social Signal Processing, 389.

Chidambaram, V., Chiang, Y. H., \& Mutlu, B. (2012). Designing persuasive robots: How robots might persuade people using vocal and nonverbal cues. Proceedings of the seventh annual ACM/IEEE international conference on human-robot interaction (pp. 293300). ACM. http://dx.doi.org/10.1145/2157689.2157798.

Choi, M., Kornfield, R., Takayama, L., \& Mutlu, B. (2017). Movement Matters: Effects of motion and mimicry on perception of similarity and closeness in robot-mediated communication. Proceedings of the 2017 CHI conference on human factors in computing systems (pp. 325-335). ACM. http://dx.doi.org/10.1145/3025453.3025734.

Cooney, S., Dignam, H., \& Brady, N. (2015). Heads first: Visual aftereffects reveal hierarchical integration of cues to social attention. PLoS One, 10(9), e0135742. http://dx. doi.org/10.1371/journal.pone.0135742.

Dillard, J. P., \& Shen, L. (2005). On the nature of reactance and its role in persuasive health communication. Communication Monographs, 72(2), 144-168. http://dx.doi. org $/ 10.1080 / 03637750500111815$.

Eyssel, F., \& Hegel, F. (2012). (S) he's got the look: Gender stereotyping of robots. Journal of Applied Social Psychology, 42(9), 2213-2230. http://dx.doi.org/10.1111/j.15591816.2012.00937.x.

Ghazali, A. S., Ham, J., Barakova, E. I., \& Markopoulos, P. (2017). Pardon the rude robot: Social cues diminish reactance to high controlling language. IEEE international symposium on robot and human interactive communication (pp. 411-417). IEEE. http://dx. doi.org/10.1109/ROMAN.2017.8172335.

Glass, G. V., Peckham, P. D., \& Sanders, J. R. (1972). Consequences of failure to meet assumptions underlying the fixed effects analyses of variance and covariance. Review of Educational Research, 42(3), 237-288. http://dx.doi.org/10.3102/ 00346543042003237.

Ham, J., Cuijpers, R. H., \& Cabibihan, J. J. (2015). Combining robotic persuasive strategies: The persuasive power of a storytelling robot that uses gazing and gestures. International Journal of Social Robotics, 7(4), 479-487. http://dx.doi.org/10.1007/ s12369-015-0280-4.

Harwell, M. R., Rubinstein, E. N., Hayes, W. S., \& Olds, C. C. (1992). Summarizing Monte Carlo results in methodological research: The one-and two-factor fixed effects ANOVA cases. Journal of Educational Statistics, 17(4), 315-339. http://dx.doi.org/10. 3102/10769986017004315.

Jacobs, R. S. (2016). Play to win Over: Effects of persuasive games. Psychology of Popular Media Culture.http://dx.doi.org/10.1037/ppm0000124.

Johnson, B. T., \& Eagly, A. H. (1989). Effects of involvement on persuasion: A metaanalysis. Psychological Bulletin, 106(2), 290. 
Lawson, J., \& Semwal, S. K. (2016). Implementing elements of fear invoking anxiety using a game platform. International conference on articulated motion and deformable objects (pp. 117-124). Springer. http://dx.doi.org/10.1007/978-3-319-41778-3_12.

Lee, K. C., Lee, S., \& Hwang, Y. (2014). The impact of hyperlink affordance, psychological reactance, and perceived business tie on trust transfer. Computers in Human Behavior, 30, 110-120. http://dx.doi.org/10.1016/j.chb.2013.08.003.

Li, C. Y. (2013). Persuasive messages on information system acceptance: A theoretical extension of elaboration likelihood model and social influence theory. Computers in Human Behavior, 29(1), 264-275. http://dx.doi.org/10.1016/j.chb.2012.09.003.

Lim, S., \& Reeves, B. (2009). Being in the game: Effects of avatar choice and point of view on psychophysiological responses during play. Media Psychology, 12(4), 348-370. http://dx.doi.org/10.1080/15213260903287242.

Lim, S., \& Reeves, B. (2010). Computer agents versus avatars: Responses to interactive game characters controlled by a computer or other player. International Journal of Human-computer Studies, 68(1), 57-68. http://dx.doi.org/10.1016/j.ijhcs.2009.09. 008.

Lix, L. M., Keselman, J. C., \& Keselman, H. (1996). Consequences of assumption violations revisited: A quantitative review of alternatives to the one-way analysis of variance $\mathrm{F}$ test. Review of Educational Research, 66(4), 579-619. http://dx.doi.org/10.3102/ 00346543066004579.

Lopez, A., Ccasane, B., Paredes, R., \& Cuellar, F. (2017). Effects of using indirect language by a robot to change human attitudes. Proceedings of the companion of the 2017 ACM/ IEEE international conference on human-robot interaction (pp. 193-194). ACM. http:// dx.doi.org/10.1145/3029798.3038310.

Louwerse, M. M., Graesser, A. C., Lu, S., \& Mitchell, H. H. (2005). Social cues in animated conversational agents. Applied Cognitive Psychology, 19(6), 693-704. http://dx.doi. org/10.1002/acp.1117.

Martin, C. D. (1997). The media Equation: How people treat computers, television and new media like real people and places. Spectrum, IEEE, 34(3), 9-10 [Book review].

Mittal, B. (1989). Measuring purchase-decision involvement. Psychology and Marketing, 6(2), 147-162.

Oreg, S., \& Sverdlik, N. (2014). Source personality and persuasiveness: Big Five predispositions to being persuasive and the role of message involvement. Journal of Personality, 82(3), 250-264. http://dx.doi.org/10.1111/jopy.12049.

Quick, B. L., \& Considine, J. R. (2008). Examining the use of forceful language when designing exercise persuasive messages for adults: A test of conceptualizing reactance arousal as a two-step process. Health Communication, 23(5), 483-491. http://dx.doi. org/10.1080/10410230802342150.

Quick, B. L., \& Stephenson, M. T. (2007). The Reactance Restoration Scale (RRS): A measure of direct and indirect restoration. Communication Research Reports, 24(2), 131-138. http://dx.doi.org/10.1080/08824090701304840.

Rains, S. A., \& Turner, M. M. (2007). Psychological reactance and persuasive health communication: A test and extension of the intertwined model. Human Communication Research, 33(2), 241-269. http://dx.doi.org/10.1111/j.1468-2958. 2007.00298.x.

Roubroeks, M., Ham, J., \& Midden, C. (2011). When artificial social agents try to persuade people: The role of social agency on the occurrence of psychological reactance. International Journal of Social Robotics, 3(2), 155-165. http://dx.doi.org/10.1007/ s12369-010-0088-1.

Roubroeks, M., Midden, C., \& Ham, J. (2009). Does it make a difference who tells you what to do?: Exploring the effect of social agency on psychological reactance. Proceedings of the 4th international conference on persuasive technology (pp. 15). ACM. http://dx.doi.org/10.1145/1541948.1541970.

Thimmesch-Gill, Z., Harder, K. A., \& Koutstaal, W. (2017). Perceiving emotions in robot body language: Acute stress heightens sensitivity to negativity while attenuating sensitivity to arousal. Computers in Human Behavior, 76, 59-67. http://dx.doi.org/10 1016/j.chb.2017.06.036.

Walter, Z., \& Lopez, M. S. (2008). Physician acceptance of information technologies: Role of perceived threat to professional autonomy. Decision Support Systems, 46(1) 206-215. http://dx.doi.org/10.1016/j.dss.2008.06.004.

van Wijngaarden, B., Schene, A. H., Koeter, M., Vázquez-Barquero, J. L., Knudsen, H. C., Lasalvia, A., et al. (2000). Caregiving in schizophrenia: Development, internal consistency and reliability of the involvement evaluation questionnaire-european version. The British Journal of Psychiatry, 177(39), 21-27. http://dx.doi.org/10.1192/ bjp.177.39.s21.

Yang, Y., Tian, Y., Fang, J., Lu, H., Wei, K., \& Yi, L. (2017). Trust and deception in children with autism spectrum disorders: A social learning perspective. Journal of Autism and Developmental Disorders, 47(3), 615-625. http://dx.doi.org/10.1007/ s10803-016-2983-2.

\section{Web reference}

Smoothie Maker. http://www.sproutonline.com/games/smoothie-maker/Accessed 10 March 2016. 